\title{
Differential Diagnosis in Children with Autistic Symptoms and Subthreshold ADOS Total Score: An Observational Study
}

\author{
Andrea De Giacomo (D) \\ Francesco Craig ${ }^{2}$ \\ Giuseppina Palermo ${ }^{3}$ \\ Annamaria Coppola' \\ Mariella Margari' \\ Stella Campanozzi' \\ Lucia Margari' \\ Marco Turi ${ }^{3}$ \\ 'Child Neuropsychiatry Unit, Department \\ of Basic Medical Sciences, Neuroscience \\ and Sense Organs, University of Bari "Aldo \\ Moro", Bari, Italy; ${ }^{2}$ Department of \\ Cultures, Education and Society, University \\ of Calabria, Rende, Italy; ${ }^{3}$ Fondazione Stella \\ Maris Mediterraneo, Potenza, Italy
}

Background: Children with autism spectrum disorder (ASD) share some symptoms with children with other neurodevelopmental disorders (ie, intellectual disability or communication disorders or language disorders). These similarities can make difficult to obtain an accurate diagnosis, which is essential to give targeted treatments to the patients. We aim to verify in our study if children with autistic traits who undergo to Autism Diagnostic Observation Schedule had specific clinical diagnosis.

Patients and Methods: We selected 73 children tested with ADOS-G or ADOS-2, for the presence of autistic symptoms. The whole sample did not reach the cut-off of ADOS and did not receive the ASD diagnosis, according to DSM-5.

Results: Results of this study showed that in order of frequency and early diagnosis, communication disorders (CD), mild intellectual disability (mID) and the attention deficit hyperactivity disorders (ADHD) represent the most common final clinical diagnosis in children with autistic traits.

Conclusion: Our results showed as the $\mathrm{CD}$ was the common diagnosis of these children and that often associated with younger age. Moreover, analyses of ADOS domains and the difference of individual items between groups did not show the capacity to differentiate between different neurodevelopmental disorders in terms of differential diagnosis, and this confirms the need for integrating multiple sources of information during the diagnostic process.

Keywords: autistic traits, ADOS, ASD, neurodevelopmental disorders, diagnosis

\section{Introduction}

Autism spectrum disorder (ASD) is a neurodevelopmental disorder characterized by difficulty with social communication and repetitive, restricted pattern of behaviors. ${ }^{1}$ The frequency of ASD diagnoses has increased dramatically in the last decade. Once considered rare, the current estimates of prevalence suggest that 1:45 in children in the United States $(2.2 \%)$ is diagnosed with an ASD. ${ }^{2,3}$ Children with ASD share some symptoms with children with other neurodevelopmental disorders (ie, intellectual disability or communication disorders or language disorders). These similarities can make difficult to obtain an accurate diagnosis, which is essential to give targeted treatments to the patients. Clinical studies between children with ASD and children with others neurodevelopmental disorders demonstrate that the first ones have a more severe impairment in joint attention, imitation skills, empathic responding, shared enjoyment, eye contact, and relationship with peers. ${ }^{4,5}$
Correspondence: Francesco Craig Department of Cultures, Education and Society, University of Calabria, Rende, Italy

Email francesco.craig@unical.it 
Differential diagnosis between ASD and other neurodevelopmental disorders (NDDs) can be difficult in children with speech delay or global developmental delay who show a certain impairment of social skills typical of ASD children. Nevertheless, children with ASD use a minor variability in conventional gestures (ie, nodding, shaking head), but they show major echolalia and the use of stereotyped language when present, are less interested in starting or answering to verbal communication and have a more severe impairment of symbolic play. Some studies have found that impairment of sensorial processing can be used to differentiate ASD from other disorders, ${ }^{6}$ but other studies have not supported this hypothesis. ${ }^{7}$ Moreover, the differential diagnosis process between ASD and other neurodevelopmental disorders can be made even more difficult by the presence clinical features shared with other diseases and comorbidities, especially in youngest children. ${ }^{8}$ This can depend on the fact that the clinical symptoms of children with neurodevelopmental delay usually undergo changes related to growth and neuronal maturation. ${ }^{9}$ The diagnostic evaluation of ASD with other NDDs could be difficult, especially in infants and toddlers as the patterns of symptomatology. ${ }^{10}$ For example, it has been suggested that ASD and ADHD are different expressions of a unique condition, each with its own distinctive features and age of onset. In this view, ADHD could manifest with traits of ASD, and ASD could manifest with traits of ADHD or other disorders. ${ }^{11,12}$ Similarly, Communication Disorder (CD) includes aspects that overlap with ASD, including trouble modifying their communication (tone of voice, pitch, and volume), which can complicate diagnosis. However, affection status, rates of social interaction impairment and restricted interests, and repetitive behavior are much lower than the ASD. ${ }^{13}$ A study using spontaneously elicited language patterns revealed that children with specific language impairment (SLI) had significantly longer speech responses than those with high-functioning autism (HFA), showing congruent, interlocutor-facing speech content similar to that of typically developing children. In contrast, children with HFA tended to show difficulty in maintaining focus on the topic of conversation, digressing much more often and exhibiting intrusive thoughts. Suggesting that this could be one of the features that differentiates SLI from HFA. ${ }^{14}$

According to the most recent prevalence study conducted by the Centers for Disease Control (CDC), 38\% of the children with ASD had an intellectual disability (ID). The comorbid condition between ASD and ID is a much higher risk factor for mental health than the two conditions in isolation. Subjects with comorbid ASD and ID show marked deficits in adaptive and functional abilities including social skills and planning. ${ }^{15}$

The issues and complexities associated with diagnosing ASD and other NDDs is an open question given the mounting evidence that different conditions could show symptomatic overlaps making diagnosis difficult particularly at an early age. Hence, the problematics of differential diagnosis remain critical for clinical approaches to autism. The Autism Diagnostic Observation Schedule (ADOS) is considered "gold standard" assessment measures in the evaluation of ASD. It is a semi-structured, standardized assessment of communication, social interaction, play, and restricted and repetitive behaviors. Several studies have contributed to building such a tool with reliable and solid metric qualities, increasing the validity and diagnostic reliability for evaluations of children with ASD. ADOS has good psychometric qualities, with a high level of reliability, internal consistency and validity confirmed by studies conducted in different countries showing crosscultural diagnostic accuracy. ${ }^{16-18}$

Previous studies showed that the ADOS is a good tool to differentiate ASD children from non-ASD children. ${ }^{4,5}$ It has been shown that among the factors that guide clinicians in the differential diagnosis between ASD and intellectual disability in school-aged children, are measures of social interaction and stereotyped behavior. ${ }^{19}$ Meanwhile, among school-age children between 6 and 15 years of age with intellectual disability who were evaluated for suspected ASD, certain characteristics such as social interaction, language, pretend play and stereotyped behaviour were able to discriminate between those with and without ASD. ${ }^{20}$ In a study by Ventola and colleagues, ADOS was used to assess the differential diagnosis between ASD and CD. In this study, children aged 12-32 months, who showed a suspect of ASD as measured by the M-CHAT (Modified Checklist for Autism in Toddlers), were assessed for a differential diagnosis between ASD, CD or global developmental delay. Results showed that subjects with ASD had atypical reciprocal social interaction, nonverbal communication and abnormal sensory responses compared to subjects with $\mathrm{CD}$ or global developmental delay. ${ }^{21}$ However, no previous study has investigated if ADOS can also evaluate the presence of other neurodevelopmental conditions, or behavioral, emotional, and cognitive disorders. Therefore, the first aim of the study was to clinically describe a sample of children with 
subthreshold ASD symptoms and ADOS total score. The second aim was to characterize this sample of children using ADOS domains and items.

\section{Method \\ Participants}

We recruited 73 children of both sexes, aged 0-18 years, consecutively referred to the Child Neuropsychiatric Operative Unit, University Hospital of Bari, and to the Stella Maris Foundation Mediterraneo of Matera in the period between start of 2019 until the beginning of 2020 and for which clinical evaluation was requested for parents' or pediatrician's concerns because of the presence of autistic traits, defined as ASD symptoms and signs (language delay, scarce joint attention, repetitive activities or restricted interests, impairment in social interactions with peers). Other inclusion criteria were ADOS total score under the cut-off for autism, and a final diagnosis different from ASD. Children were excluded from participation in this study if they had neurological disorders of known etiology; major physical abnormalities; serious head injury; or history of psychotropic drugs assumption at the moment of clinical evaluation.

\section{Procedure}

All children underwent a comprehensive clinical assessment, including anamnestic interview, physical and neurological examination, and laboratory and instrumental tests when indicated according to clinical suspicion.

They all received a full neuropsychiatric assessment, including the ADOS administration, carried out by psychologists and child and adolescent neuropsychiatrists from both recruiting centers, who had specific training in ADOS administration and clinical expertise in neurodevelopmental disorders. After the exclusion of ASD diagnosis according to Diagnostic and Statistical Manual of Mental Disorders, Fifth Edition (DSM-5) criteria, clinical evaluation went forward and they were supported by the administration of other neuropsychological standardized tools, chosen according to clinical judgment. All the evaluations were discussed in regular reliability meetings, supervised by senior researchers, and psychiatric diagnosis were made according to DSM-5 criteria.

This study has been approved by the Local Ethics Committee of Azienda Ospedaliero-Universitaria Policlinico di Bari (id cod. CFDADG). This observational study was conducted in accordance with the ethical standards of Helsinki Declaration; written informed consent from a parent/guardian of each participant was obtained.

\section{Measures}

Autism Diagnostic Observation Scale (ADOS-G and ADOS-2)

ADOS-G and ADOS-2 comprise standardised activities that allow the observation of peculiar behaviours in order to apply a diagnosis of ASD. They assess in particular aspects related to communication, social interaction, play and imitative behaviour. The ADOS observation takes about 45 minutes and the total score obtained at the end of the assessment, in the two domains social affect and restricted and repetitive behavior, makes it possible to define whether a subject is on the autistic spectrum. It consists of four different modules that are chosen according to the level of expressive language shown by the child.

In this study, ADOS- $\mathrm{G}^{22}$ and ADOS- $2^{23}$ were applied. Both have been used as inclusive/exclusive criteria for the patients in our study: only patients with a total score (AS + CRR) inferior to the cut-off for the autistic spectrum that at the ending of the evaluation did not receive an Autistic Spectrum Disorder diagnosis have been selected. Moreover, according to age and level of development of our sample, only modules 1, 2 and 3 of ADOS-2 have been taken into consideration. In line to published algorithms, ${ }^{24,25}$ the Calibrated Severity Score (CSS) was calculated for each participant using the Total, Social Affect (SA) and Restricted Repetitive Behaviors (RRB) scores. The CSS range is $1-10$ and makes it possible to compare different versions and modules of ADOS, providing also a measure of autism symptoms that is age and language-independent and thereby more suitable than ADOS scores for assessing ASD severity. ${ }^{26}$ ADOS-G scores were first converted to ADOS-2 scores on the basis of the new algorithm proposed by Gotham et al. ${ }^{27}$ To calculate the CSS for the Toddler Module, we have applied the algorithm proposed by Esler et $\mathrm{al}^{28}$ in order to facilitate comparison with the other modules of ADOS-2.

\section{Cognitive and Neuropsychological Assessment}

Given the differences in the verbal skills and level of functioning of the children recruited for the study, different standardized tests were used to assess the intelligence quotient (IQ). These included the Leiter International Performance Scale-Revised ${ }^{29}$ (in nonverbal children), and the Italian version of Wechsler Preschool and Primary 
Scale of Intelligence ${ }^{30}$ or the Wechsler Intelligence Scale for Children. ${ }^{31}$

\section{Other Standardized Tools}

After exclusion of ASD diagnosis, all children were assessed, according to clinical indication, with specific standardized tests in order to support an appropriate diagnosis (eg Vineland Adaptive Behavior Scales (VABS), Swanson, Nolan, and Pelham-IV Questionnaire (SNAPIV), Conners Rating Scales, etc). The scores obtained by these tools were not reported in this study, neither included in statistical analysis, because of the low sample size.

\section{Statistical Approach}

We preliminarily examined that all continuous variables were normally distributed using the skewness and the Kolmogorov-Smirnov test. The descriptive analyses and chi-square analysis was used for categorical and continuous independent variable. One-way analysis of variance (ANOVA) was performed to evaluate differences in IQ and age among all groups. The analysis of ADOS total scores was carried out using a MANCOVA and controlling for those variables that were statistically different in the different groups (eg, age and IQ). In order to compare ADOS scores across all groups we examined the presence or absence of behavioral abnormalities. The scores of the ADOS items were converted to 0 when the absence of symptoms was detected, while scores 1, 2, 3 were converted to 1 , indicating the presence of symptoms. This allowed us to directly compare frequency of atypical behaviors across groups. Comparable items from different ADOS modules were pooled, as suggested by Lord et $\mathrm{al}^{22}$ and as indicated in Table 1. The proportions of scores of 1 (atypical behavior) and 0 (absence of abnormality) in children who did not receive the ASD diagnosis for each pooled item were assessed and compared across groups, using Chi-square analysis of the distribution of atypical behavior. The data were analyzed using SPSS Statistics, version 20.0 (IBM Corp., NY, USA).

\section{Results}

The main sample taken into examination consists of 73 children between 2 years and 7 months old and 13 years with an mean age of 5.83 (2.53) years, of which 12 were females (14\% of the sample) and 61 were males $(86 \%$ of the sample). All these patients received one or more diagnosis for neurodevelopmental disorders, different than ASD because under the ADOS cut-off score and clinically according to DSM-5 they only had autistic traits regrouped into three principal diagnoses: 1) communication disorders (CD) $(n=44$, representing $60 \%$ out of the whole children, 8 females and 36 males)included different subsamples as follows: 28 children affected by language disorder: 9 children with phonetic/phonological disorder and 7 children with social communication disorder; 2) mild intellectual disability ( $\mathrm{n}=18,24 \%$ of total sample, 2 females and 16 males); 3) ADHD ( $\mathrm{n}=11,15 \%$ of the whole sample, 1 females and 10 males). All demographic and clinical data of the ADHD, CD, mID group are summarized in Table 2.

Looking at differences between groups, one-way ANOVA indicated a significant difference on age among the groups $\left(F_{(2,72)}=5.18 \mathrm{p}=0.008\right)$; Bonferroni post hoc test revealed that the difference was due to the younger age of the CD compared to ADHD group ( $p=0.03$ ); no differences were found between ADHD and mID group $(\mathrm{p}>0.05)$. One-way ANOVA showed also a significant difference on total IQ among the groups $\left(F_{(2,72)}=30.41\right.$ $\mathrm{p}<0.0001)$; Bonferroni post hoc test revealed that the difference was lower IQ of the mID group compared to ADHD $(p<0.0001)$ and CD groups; no differences were found between ADHD and CD group ( $p>0.05$ ). Chi-square analysis did not reveal a significant difference on gender distribution $\left(\chi^{2}(2)=0.86, p=0.65\right)$, comorbidity $\left(\chi^{2}(2)=0.94\right.$, $\mathrm{p}=0.62)$ and language presence $\left(\chi^{2}(2)=2.99, \mathrm{p}=0.22\right)$ among $\mathrm{CD}$, ADHD and mID groups.

\section{ADOS Domain and Item Analyses}

The distribution of ADOS modules in the different groups shows that the percentage of Module $1(45.5 \%)$ was significantly higher (Table 2) in the $\mathrm{CD}$ group if compared with the other two groups. In the ADHD group we only used modules 2 and 3 in equal quantity, respectively, $54.5 \%$ and $45.5 \%$. In the mID group, module $2(61.1 \%)$ was mostly used, "created for children who produce language for complete phrases, even if not fully fluent, even if they are less than 30 months old". To determine whether children among groups showed difference across ADOS domains, we performed a one-way MANCOVA (controlling for IQ, given the difference between groups and presence or absence of comorbidity) that did not reveal a statistically significant difference among group in summary score of ADOS domains, Social Affect $\left(\mathrm{SA} F_{(4,134)}\right.$ $=1.28 \mathrm{p}=0.28$ ), Restricted and Repetitive Behavior (RRB $\left.F_{(4,134)}=0.34 \mathrm{p}=0.71\right)$ and Total Score $F_{(4,134)}=0.84$ $\mathrm{p}=0.43$ ). In order to control evaluate the presence of comorbidities as a confounding factor, we performed 
Table I Modules I-3: Distribution of Items Across Modules

\begin{tabular}{|l|c|c|c|}
\hline & $\begin{array}{c}\text { Module I Preverbal/Single } \\
\text { Words/Simple Phrases }\end{array}$ & $\begin{array}{c}\text { Module 2 Flexible } \\
\text { Phrase Speech }\end{array}$ & $\begin{array}{c}\text { Module 3 Fluent Speech } \\
\text { Child/Adolescent }\end{array}$ \\
\hline Pointing & A7 & A6 & - \\
Gestures & A8 & A7 \\
Eye contact & BI & BI & BI \\
Facial expression & B3 & B2 & B2 \\
Shared enjoyment & B5 & B3 & B4 \\
Showing & B9 & BI2 & A7 \\
Spontaneous initiation of joint attention & BI0 & B6 & BI0 \\
Response to joint attention & BII & BII & A4 \\
Quality of social overtures & BI2 & A4 & DI \\
Stereotyped/idiosyncratic words or & A5 & DI & D2 \\
phrases & & D2 & D4 \\
Unusual sensory behaviors & DI & D4 & \\
Mannerisms & D2 & & \\
Repetitive interests and & D4 & & \\
behaviors & & & \\
\hline
\end{tabular}

a one-way MANCOVA, controlling for IQ and presence or absence of comorbidity, which did not reveal a statistically significant difference among group in summary score of ADOS domains, Social Affect $\left(\mathrm{SA} F_{(4,134)}=1.28 \mathrm{p}=0.28\right)$, Restricted and Repetitive Behavior $\left(\operatorname{RRB} F_{(4,134)}=0.34\right.$ $\mathrm{p}=0.71)$ and Total Score $\left.F_{(4,134)}=0.84 \mathrm{p}=0.43\right)$ confirming the previous analysis. To compare single ADOS items between groups item scores were converted to 0 when the absence of symptoms was detected, while scores 1, 2, 3 were converted to 1 , indicating the presence of symptoms as showed in Table 3 and in Figure 1. However, Chisquare analysis of the distribution of atypical behavior among groups did not reveal a significant difference across all ADOS items (all $\mathrm{p}$ values $>0.05$ ).

\section{Discussion}

This manuscript describes a naturalistic study exploring the characteristics of those who score below threshold on the ADOS and do not receive a full ASD diagnosis. The results of this study highlight that children with suspected ASD (for the presence of "autistic symptoms"), who did not reach the ADOS-G or ADOS-2 cut-off for autism and who did not received a full diagnosis of ASD according to DSM-5 criteria received a main diagnosis of another neurodevelopmental disorder among $\mathrm{CD}, \mathrm{mID}$, and ADHD. Particularly, as expected, the most represented disorder was $\mathrm{CD}$, identified as main diagnosis in $60 \%$ of the cases and mostly represented by language disorder (with both expressive and receptive functions compromised), as demonstrated above, one of the most common reasons for suspecting ASD is a delay in language development. ${ }^{32}$

The other two other clinical categories diagnoses were identified in lower percentage of cases and were represent by "mild intellectual disability" (24\%) and externalizing problems (16\%), mostly represented by "attention deficit disorder with hyperactivity". These results are consistent with the finding that child's language delay and/or deficit in social communication are the main warning signs for ASD, followed in the order by opposition, hyperactivity, lack of attention, and delay in reaching motor milestones, among others. ${ }^{33}$ Really, these possibilities of differential diagnosis should be always kept in mind when evaluating children with suspected ASD, mainly when ADOS score is subthreshold for autism.

Age of the patients is one of the variables that show a significant statistical difference between the three groups: children with communication disorder are younger (5.14 years) than children with intellectual disability (6.74), and than children with ADHD (7.9 years). This is confirmed by the Simms study, ${ }^{34}$ according to which before the entire development of linguistic, receptive and expressive skills ( $<4$ years), children with a language disorder are likely to look like those with autism spectrum disorder; for example, they may not be able to interact 
Table 2 Demographic, IQ and ADOS-CSS Scores Among All Groups

\begin{tabular}{|c|c|c|c|c|c|}
\hline & $\begin{array}{l}\text { Communication } \\
\text { Disorders }\end{array}$ & $\begin{array}{l}\text { Intellectual } \\
\text { Disability }\end{array}$ & ADHD & $X^{2}$ or $F p$ value & Total \\
\hline Age (years) & $5.14(2.0)$ & $6.74(2.7 \mathrm{I})$ & $7.27(3.05)$ & $\begin{array}{c}F_{(2,72)}=5.18 \\
p=0.008\end{array}$ & - \\
\hline Female sex & $8(18.2 \%)$ & $2(11.1 \%)$ & I (9.1\%) & $\begin{array}{c}\chi^{2}(2)=0.86 \\
p=0.65\end{array}$ & $\begin{array}{c}12 \\
(15.1 \%)\end{array}$ \\
\hline IQ Total & 96.41 (16.6I) & $65.94(4.33)$ & $\begin{array}{l}101.54 \\
(18.86)\end{array}$ & $\begin{array}{c}F(2,72)=30.41 \\
p<0.000 I\end{array}$ & - \\
\hline \multicolumn{6}{|l|}{ IQ classification } \\
\hline Normal & 33 (75.0\%) & $0(0 \%)$ & 9 (8I.8\%) & & $\begin{array}{c}42 \\
(57.5 \%)\end{array}$ \\
\hline Borderline & $8(18.2 \%)$ & $2(11.1 \%)$ & I (9.1\%) & & $\begin{array}{c}11 \\
(15.1 \%)\end{array}$ \\
\hline ID & $3(6.8 \%)$ & 16 (88.9\%) & I (9.1\%) & & $\begin{array}{c}20 \\
(27.4 \%)\end{array}$ \\
\hline Non-verbal & 7 (15.9\%) & I (5.6\%) & $0(0 \%)$ & $\begin{array}{l}\chi^{2}(2)=2.99 \\
p=0.223\end{array}$ & $8(11 \%)$ \\
\hline Comorbidity & $21(44.7 \%)$ & II (6I.I\%) & $6(54.5 \%)$ & $\begin{array}{l}\chi^{2}(2) 0.94 \\
p=0.622\end{array}$ & $\begin{array}{c}38 \\
(52.1 \%)\end{array}$ \\
\hline \multicolumn{6}{|l|}{ Module } \\
\hline I & 20 (45.5\%) & $5(27.8 \%)$ & $0(0 \%)$ & & $\begin{array}{c}25 \\
(34.2 \%)\end{array}$ \\
\hline 2 & $20(45.5 \%)$ & II (6I.I\%) & $6(54.5 \%)$ & & $\begin{array}{c}37 \\
(50.7 \%)\end{array}$ \\
\hline 3 & $4(9.1 \%)$ & $2(11.1 \%)$ & $5(45.5 \%)$ & & $\begin{array}{c}11 \\
(15.1 \%)\end{array}$ \\
\hline Social affect (SA) & $3.55(1.83)$ & $3.67(1.78)$ & $2.55(1.63)$ & $\begin{array}{c}F_{(2,70)}=0.92 \\
p=0.40\end{array}$ & \\
\hline $\begin{array}{l}\text { Repetitive and restricted behaviour } \\
\text { (RRB) }\end{array}$ & $0.93(0.97)$ & $0.83(0.78)$ & $1.0(1.18)$ & $\begin{array}{c}F_{(2,70)}=0.23 \\
p=0.79\end{array}$ & \\
\hline Total ADOS-score & $4.48(2.08)$ & $4.5(2.03)$ & $3.5(1.69)$ & $\begin{array}{c}F_{(2,70)}=0.38 \\
P=0.68\end{array}$ & \\
\hline
\end{tabular}

Notes: Data are reported as frequency (proportions (\%)) or mean (SD). Chi-square or ANOVA results are reported. Significant comparisons are highlighted in bold $(\mathrm{p}<0.05)$.

with strangers, show a lack of eye contact, be stuck in highly structured routines and show difficulties in adapting when they are in new settings and environments.

In a recent study, Charman et $\mathrm{a}^{35}$ investigated ADOS outcome in low-risk children, with no family history of ASD, involved in surveillance protocol to detect early sign of autism. Authors found that $15 \%$ of low-risk children showed an elevated autism symptomatology, using an arbitrary cut-off ADOS-CSS score, but they do not receive a diagnosis of autism at 36 months of age. Charman et al suggest that these high ADOS scores could be the expression of behavioural characteristics not related to ASD, such as anxiety and social inhibition and hyperactivity, which could result in high ADOS scores. Our results are in line with the Charman et al study, since our children arrived for evaluation for suspected autism spectrum 
Table 3 Distribution of Atypical Behavior (Score of I or More) Across Groups

\begin{tabular}{|c|c|c|c|c|}
\hline & $\begin{array}{l}\text { Communication Disorders } \\
\qquad(n=44)\end{array}$ & $\begin{array}{l}\text { Intellectual Disability } \\
\qquad(n=18)\end{array}$ & $\begin{array}{l}\text { ADHD } \\
(n=I I)\end{array}$ & $X^{2}$ (p value) \\
\hline Pointing & $14(35)$ & $7(43.8)$ & $2(33.3)$ & $\chi^{2}(2)=0.4 I(0.8 I)$ \\
\hline Gestures & $14(31.8)$ & $6(33.3)$ & $4(36.4)$ & $\chi^{2}(2)=0.08(0.95)$ \\
\hline Eye contact & $6(13.6)$ & $3(16.7)$ & $2(18.6)$ & $\chi^{2}(2)=0.19(0.90)$ \\
\hline Facial expression & $17(38.6)$ & 7 (38.9) & $2(18.2)$ & $\chi^{2}(2)=1.7 \mid(0.42)$ \\
\hline Shared enjoyment & $9(20.5)$ & $3(16.7)$ & I (9.1) & $\chi^{2}(2)=0.7(0.67)$ \\
\hline Showing & $15(34.1)$ & $9(50)$ & $3(27.3)$ & $\chi^{2}(2)=1.91(0.38)$ \\
\hline Spontaneous initiation of joint attention & $10(22.7)$ & $3(16.7)$ & $2(18.2)$ & $\chi^{2}(2)=0.33(0.84)$ \\
\hline Response to joint attention & $14(31.8)$ & $7(38.9)$ & $5(45.5)$ & $\chi^{2}(2)=0.82(0.66)$ \\
\hline Quality of social overtures & $19(43.2)$ & $6(33.3)$ & $2(18.2)$ & $\chi^{2}(2)=2.49(0.28)$ \\
\hline $\begin{array}{l}\text { Stereotyped/idiosyncratic words or } \\
\text { phrases }\end{array}$ & $5(11.4)$ & $2(11.2)$ & $0(0)$ & $\chi^{2}(2)=1.37(0.50)$ \\
\hline Unusual sensory behaviors & $7(15.9)$ & $4(22.2)$ & I (9.1) & $\chi^{2}(2)=0.88(0.64)$ \\
\hline Mannerisms & $11(25)$ & $3(16.7)$ & $3(27.3)$ & $\chi^{2}(2)=0.61 \quad(0.77)$ \\
\hline Repetitive interests and behaviors & $13(29.5)$ & $6(33.3)$ & $5(45.5)$ & $\chi^{2}(2)=1.91(0.38)$ \\
\hline
\end{tabular}

Notes: Data are frequency (proportions (\%)).

disorder presenting high levels of autistic traits below the cut-off for a diagnosis. The elevated autistic traits in these children could be, as suggested, an expression of other behavioral features (eg, ADHD, anxiety, communication disorder) not related to an autism spectrum disorder, but as a sign of symptom overlapping between other neurodevelopmental disorders.

Another clinical characteristic that can help us formulate a diagnosis is a direct consequence of everything we said until now. Using module 1 (that was built for children older than 31 months who still did not develop fluent language), points us, for age and language level, towards a communication disorder. Using module 2 (that was built for children producing language with complete sentences, even if not completely fluent, even if younger than 30 months), points us towards an intellectual disability but also towards ADHD, while using module 3 (made for children and young adolescents with fluent verbal language) points us towards an ADHD diagnosis, according to age and development level. As mentioned above, previous studies showed that the ADOS is a good tool to differentiate ASD children from non-ASD children. Most of the studies on ASD subthreshold were done on adults with other diagnoses: mood disorders, personality disorders, DOC disorders and eating behavior disorders. ${ }^{36}$ However, in children, there are no studies regarding the utility of the ADOS in orienting differential diagnosis between NDDs different from autism, but most concern the comorbidity between autism with other psychiatric disorders. ${ }^{37}$ There is one study about Social Responsiveness Scale (SRS) in children with subthreshold ASD symptoms, but no alternative diagnosis has been formulated ${ }^{38}$ As suggested by Lord et $\mathrm{al}^{22}$ our results confirm the need for multiple sources of information during the diagnostic process. Given that a significant percentage of children with mental disorders not related to autism have been misclassified as having ASD, it is important to not interpret ADOS scale scores in an absolute and isolated way but to always integrate them with other information deriving also from the different contexts of the subject's life (teachers, caregivers). The use of collateral information seems particularly important when assessing children with suspected autism. This study gives us a foundation to expand our research in the autistic traits field, in children that do not reach the diagnostic criteria to qualify as autistic spectrum disorder. For this purpose, it could be useful to expand the total sample and to have a greater homogeneity in the diagnostic groups, besides elaborating the statistical analysis on the single ADOS sub items rather than on the sums of points per area, since a small sample size is one of the major limitations of this study. In fact, it neither allow us to stratify children by age in each subgroup nor to study the predictive value of symptoms. It would also be interesting to follow the diagnostic evolution of these children, in order to elucidate the stability or the reduction of their autistic traits or, on the contrary, whether a proportion of children will develop a full ASD 


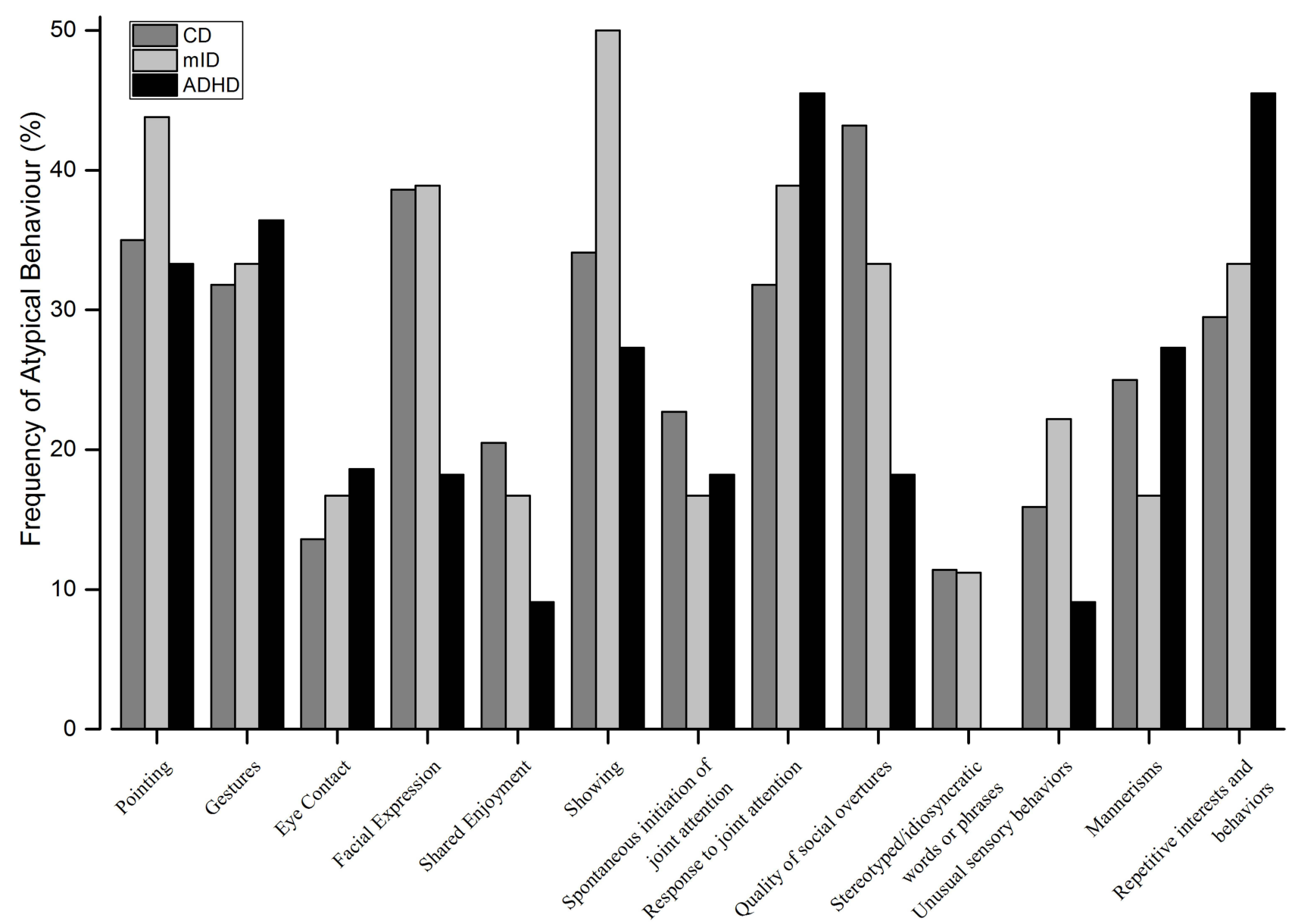

Figure I Frequency of atypical behavior for the ADOS single items across different diagnostic groups (communication disorder (CD), mild intellectual disability, ADHD).

diagnosis over the years. Further studies with a larger sample size, matched for age and sex, could provide a better understanding of the predictive value of autistic symptoms, measured by ADOS, in guiding the differential diagnosis.

\section{Conclusion}

In summary, we studied behavioral and clinical profile of children who display ASD autistic traits. Our results showed the communication disorders was the most common diagnosis of these children and that often was associated with younger age. Although ADOS is useful in supporting the differential diagnosis between ASD and non-ASD, this study has outlined that it appears scarcely capable of identifying specific patterns of autistic traits useful in supporting the diagnosis of neurodevelopmental disorders other than autism. However, given the exiguity of our sample, further studies are needed to confirm these findings.

\section{Acknowledgments}

Thanks to Prof. Silvio Tafuri and Dr Valerio Giannico of Department of Biomedical Sciences and human Oncology, University of Bari "Aldo Moro", for his collaboration in statistics. We would like to thank Dr Lucia Marzulli for her contribution to this manuscript. For author M.T., the research was supported by the EU Horizon 2020 research and innovation programme under Grant Agreement N. 832813 Spatiotemporal mechanisms of generative perception - GenPercept.

\section{Disclosure}

The authors report no conflicts of interest in this work.

\section{References}

1. American Psychiatric Association. Diagnostic and Statistical Manual of Mental Disorders. 5th ed. 2013.

2. Narzisi A, Posada M, Barbieri F, et al. Prevalence of autism spectrum disorder in a large Italian catchment area: a school-based population study within the ASDEU project. Epidemiol Psychiatr Sci. 2018;29:e5. doi: $10.1017 /$ S2045796018000483 
3. Zablotsky B, Black LI, Maenner MJ, Schieve LA, Blumberg SJ. Estimated prevalence of autism and other developmental disabilities following questionnaire changes in the 2014 National Health Interview Survey. Natl Health Stat Report. 2015;(87):1-20.

4. Charman T, Swettenham J, Baron-Cohen S, Cox A, Baird G, Drew A. An experimental investigation of social-cognitive abilities in infants with autism: clinical implications. Infant Ment Health J. 1998;19 (2):260-275. doi:10.1002/(SICI)1097-0355(199822)19:2<260::AIDIMHJ12>3.0.CO;2-W

5. Noterdaeme M, Sitter S, Mildenberger K, Amorosa H. Diagnostic assessment of communicative and interactive behaviours in children with autism and receptive language disorder. Eur Child Adolesc Psychiatry. 2000;9(4):295-300. doi:10.1007/s007870070033

6. Rogers SJ, Hepburn S, Wehner E. Parent reports of sensory symptoms in toddlers with autism and those with other developmental disorders. J Autism Dev Disord. 2003;33(6):631-642. doi:10.1023/ B:JADD.0000006000.38991.a7

7. Saemundsen E, Magnusson P, Smari J, Sigurdardottir S. Autism diagnostic interview-revised and the childhood autism rating scale: convergence and discrepancy in diagnosing autism. J Autism Dev Disord. 2003;33(3):319-328. doi:10.1023/A:1024410702242

8. Okyar E, Görker I. Examining the autistic traits in children and adolescents diagnosed with attention-deficit hyperactivity disorder and their parents. BMC Psychiatry. 2020;20(1):285. doi:10.1186/ s12888-020-02703-z

9. Levy Y. 'Developmental Delay' reconsidered: the critical role of agedependent, co-variant development. Front Psychol. 2018;9:503. doi:10.3389/fpsyg.2018.00503

10. Cooper M, Martin J, Langley K, Hamshere M, Thapar A. Autistic traits in children with ADHD index clinical and cognitive problems. Eur Child Adolesc Psychiatry. 2014;23(1):23-34. doi:10.1007/ s00787-013-0398-6

11. Sokolova E, Oerlemans AM, Rommelse NN, et al. Mediation analysis of the comorbidity between Attention Deficit Hyperactivity Disorder (ADHD) and Autism Spectrum Disorder (ASD). J Autism Dev Disord. 2017;47(6):1595-1604. doi:10.1007/ s10803-017-3083-7

12. Craig F, Lamanna AL, Margari F, Matera E, Simone M, Margari L. Overlap between autism spectrum disorders and attention deficit hyperactivity disorder: searching for distinctive/common clinical features. Autism Res. 2015;8(3):328-337. doi:10.1002/aur.1449

13. Flax J, Gwin C, Wilson S, Fradkin Y, Buyske S, Brzustowicz L. Social (Pragmatic) communication disorder: another name for the broad autism phenotype? Autism. 2019;23(8):1982-1992. doi:10.1177/1362361318822503

14. Craig M, Trauner D. Comparison of spontaneously elicited language patterns in specific language impairment and high-functioning autism. Pediatr Neurol. 2018;79:53-58. doi:10.1016/j. pediatrneurol.2017.09.017

15. Dunn K, Rydzewska E, Fleming M, Cooper SA. Prevalence of mental health conditions, sensory impairments and physical disability in people with co-occurring intellectual disabilities and autism compared with other people: a cross-sectional total population study in Scotland. BMJ Open. 2020;10(4):e035280. doi:10.1136/bmjopen2019-035280

16. Matson JL, Matheis M, Burns CO, et al. Examining cross-cultural differences in autism spectrum disorder: a multinational comparison from Greece, Italy, Japan, Poland, and the United States. Eur Psychiatry. 2017;42:70-76. doi:10.1016/j.eurpsy.2016.10.007

17. Medda JE, Cholemkery H, Freitag CM. Sensitivity and Specificity of the ADOS-2 algorithm in a large German Sample. J Autism Dev Disord. 2019;49(2):750-761. doi:10.1007/s10803-018-3750-3
18. Pacifico MC, de Paula CS, Namur VS, Lowenthal R, Bosa CA, Teixeira M. Preliminary evidence of the validity process of the Autism Diagnostic Observation Schedule (ADOS): translation, cross-cultural adaptation and semantic equivalence of the Brazilian Portuguese version. Trends Psychiatry Psychother. 2019;41 (3):218-226. doi:10.1590/2237-6089-2018-0063

19. Pedersen AL, Pettygrove S, Lu Z, et al. DSM criteria that best differentiate intellectual disability from autism spectrum disorder. Child Psychiatry Hum Dev. 2017;48(4):537-545. doi:10.1007/ s10578-016-0681-0

20. Hartley SL, Sikora DM. Detecting autism spectrum disorder in children with intellectual disability: which DSM-IV-TR criteria are most useful? Focus Autism Other Dev Disabl. 2010;25(2):85-97. doi:10.1177/1088357609356094

21. Ventola P, Kleinman J, Pandey J, et al. Differentiating between autism spectrum disorders and other developmental disabilities in children who failed a screening instrument for ASD. J Autism Dev Disord. 2007;37(3):425-436. doi:10.1007/s10803-006-0177-z

22. Lord C, Risi S, Lambrecht L, et al. The autism diagnostic observation schedule-generic: a standard measure of social and communication deficits associated with the spectrum of autism. J Autism Dev Disord. 2000;30(3):205-223. doi:10.1023/A:1005592401947

23. Lord C, DiLavore PC. Autism Diagnostic Observation Schedule. 2nd ed. ADOS-2. Torrance: Western Psychological Services: 2012

24. Gotham K, Pickles A, Lord C. Standardizing ADOS scores for a measure of severity in autism spectrum disorders. J Autism Dev Disord. 2009;39(5):693-705. doi:10.1007/s10803-008-0674-3

25. Hus V, Gotham K, Lord C. Standardizing ADOS domain scores: separating severity of social affect and restricted and repetitive behaviors. $J$ Autism Dev Disord. 2014;44(10):2400-2412. doi:10.1007/s10803-012-1719-1

26. Shumway S, Farmer C, Thurm A, Joseph L, Black D, Golden C. The ADOS calibrated severity score: relationship to phenotypic variables and stability over time. Autism Res. 2012;5(4):267-276. doi:10.1002/ aur. 1238

27. Gotham K, Risi S, Pickles A, Lord C. The autism diagnostic observation schedule: revised algorithms for improved diagnostic validity. J Autism Dev Disord. 2007;37(4):613-627. doi:10.1007/s10803-0060280-1

28. Esler AN, Bal VH, Guthrie W, Wetherby A, Ellis Weismer S, Lord C. The autism diagnostic observation schedule, toddler module: standardized severity scores. J Autism Dev Disord. 2015;45(9):2704-2720. doi:10.1007/s10803-015-2432-7

29. Roid GM, Miller LJ. Leiter International Performance ScaleRevised: Examiners Manual. Wood Dale ISC, Ed; 1997

30. Wechsler D. Wechsler Preschool and Primary Scale of IntelligenceRevised. San Antonio; 1989.

31. Wechsler D. Wechsler Intelligence Scale for Children. US: Psychological Corporation; 1949.

32. De Giacomo A, Fombonne E. Parental recognition of developmental abnormalities in autism. Eur Child Adolesc Psychiatry. 1998;7 (3):131-136. doi:10.1007/s007870050058

33. Guinchat V, Chamak B, Bonniau B, et al. Very early signs of autism reported by parents include many concerns not specific to autism criteria. Res Autism Spectr Disord. 2012;6(2):589-601. doi:10.1016/j. rasd.2011.10.005

34. Simms MD, Jin XM. Autism, language disorder, and social (pragmatic) communication disorder: DSM-V and differential diagnoses. Pediatrics Rev. 2015;36(8):355-363. doi:10.1542/pir.36-8-355

35. Charman T, Young GS, Brian J, et al. Non-ASD outcomes at 36 months in siblings at familial risk for autism spectrum disorder (ASD): a baby siblings research consortium (BSRC) study. Austism Res. 2017;10(1):169-178. doi:10.1002/aur.1669 
36. Oyku Memis C, Sevincok D, Dogan B, et al. The subthreshold autistic traits in patients with adult-onset obsessive-compulsive disorder: a comparative study with adolescent patients. Riv Psichiatr. 2019;54(4):168-174. doi:10.1708/3202.31799

37. Colombi C, Fish A, Ghaziuddin M. Utility of the ADOS-2 in children with psychiatric disorders. Eur Child Adolesc Psychiatry. 2019;29 (7):989-992. doi:10.1007/s00787-019-01411-8
38. Crehan ET, Baer J, Althoff RR, Constantino JN. Tracking the influence of autistic traits on competencies among school aged children with subthreshold autistic traits: a longitudinal Study. Child Psychiatry Hum Dev. 2018;49(6):941-955. doi:10.1007/s10578-0180809-5

\section{Publish your work in this journal}

Neuropsychiatric Disease and Treatment is an international, peerreviewed journal of clinical therapeutics and pharmacology focusing on concise rapid reporting of clinical or pre-clinical studies on a range of neuropsychiatric and neurological disorders. This journal is indexed on PubMed Central, the 'PsycINFO' database and CAS, and is the official journal of The International Neuropsychiatric Association (INA). The manuscript management system is completely online and includes a very quick and fair peer-review system, which is all easy to use. Visit http://www.dovepress.com/testimonials.php to read real quotes from published authors. 